\title{
An Efficient Method of Number Plate Extraction from Indian Vehicles Image
}

\author{
Sarbjit Kaur \\ Research Scholar \\ CT Group of Institutions, Jalandhar
}

\author{
Sukhvir Kaur \\ Assistant Professor \\ CT Group of Institutions, Jalandhar
}

\begin{abstract}
Automatic Number Plate Recognition (ANPR) is an imageprocessing technology that identifies vehicles by their number plates without direct human intervention. It is an application of computer vision and important area of research due to its many applications. The main process of ANPR is divided into four stages. This paper presents a simple and efficient method for the extraction of number plate from the vehicle image based on morphological operations, thresholding and sobel edge detection, and the connected component analysis.
\end{abstract}

\section{Keywords}

Automatic Number Plate Recognition, number plate extraction, morphological operations, thresholding, sobel edge detection.

\section{INTRODUCTION}

Automatic number plate recognition (ANPR) system is important method used in Intelligent Transportation System (ITS). Vehicles play important role in transportation. Due to population growth and human needs, the use of vehicles has been increasing day by day. Therefore control of vehicles is becoming a big problem. ANPR system is one of the methods used for the effective control of these vehicles. ANPR is an image processing technology that is used to identify the vehicles by tracking their number plate without direct human intervention. There are number of applications of ANPR, such as automatic ticketing of vehicles at parking area, automatic toll collection at toll plaza, traffic monitoring, tracking vehicles during signal violation, access control in building and parking areas, border control, stolen car detection, marketing research, journey time measurement and in many other application with huge saving of human effort and cost. ANPR is also known as number plate tracking, vehicle number plate recognition, car plate recognition, automatic vehicle identification, automatic license plate reader, license plate recognition etc.

In all developed counties and in most of the developing counties the attributes of the number plate are strictly maintained. The size of number plate (aspect ratio), background color of number plate, color of characters, font style, font size, spacing between subsequent characters, scripts, number of lines in the number plate etc. are maintained very strictly. Aspect ratio is very important factor in standardized vehicle's number plates.

In almost of the developing and developed countries the aspect ratio of all vehicles' number plates is same where aspect ratio is ratio of width over height of number plate and is calculated as

\section{Aspect Ratio $=$ Width $/$ Height}

The extraction of Indian number plate is difficult compared to the foreign number plate as in India there is no standard followed for the aspect ratio of Indian number plate. This factor makes the detection and extraction of number plate and also segmentation and recognition of characters on the number plates very difficult.

There are two types of number plates used for two categories of vehicles in India. In case of commercial vehicles, the number plate has yellow background with back color characters on it. In case of private vehicles, they have white background with black character on it. The current vehicle registration scheme in India consists of two -letter code that indicate the state in which the vehicle is registered followed by two-digit numeric code that indicate the district followed by series code. This is followed by four digits actual registration number that uniquely identifies the vehicle. Figure 1 indicates the sample of an Indian vehicle number plate in which 1 represent two-letter state code, 2 represent two-digit district code, 3 represent series code, and 4 represent actual registration number that is unique for each vehicle.

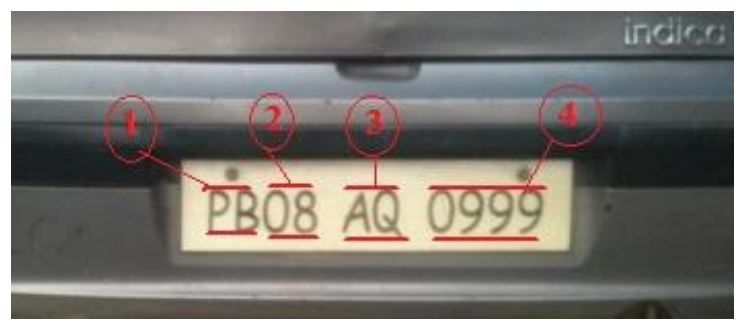

Fig 1: Sample of Indian vehicle Number Plate

The ANPR work is generally framed into the 4 stages: First stage is to acquire the image and performing some preprocessing on it; Second stage is extraction of number plate from whole body of vehicle image; Third Stage is segmentation or isolation of characters from the extracted number plate and fourth stage is to recognize the segmented characters and to display the output result. From the entire input image, only the number plate is detected and processed further in character segmentation. From the extracted number plate each character is isolated by segmentation in the character segmentation phase. After the segmentation of character the recognition is done in last character recognition phase.

ANPR system as shown in figure 2 consists of four phases:-

1) Image Acquisition and Pre-processing

2) Extraction of Number Plate

3) Character Segmentation

4) Character Recognition 


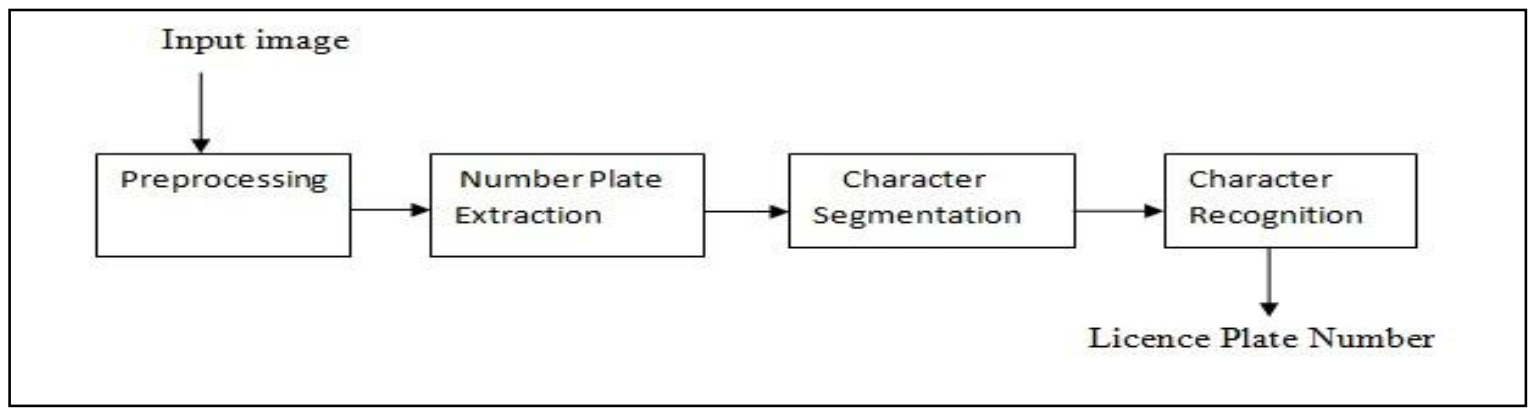

Fig 2: ANPR system

The first phase is image acquisition in which input image is acquired through camera and then some pre-processing done on it to enhance the visibility of image. Pre-processing is mainly used to improve the contrast of the image, enhance the processing speed, and to reduce the noise in the image. In order to reduce the problem of low contrast and low quality in vehicles input images, images are enhanced by using histogram equalization on gray scale image. After preprocessing the number plate area is detected and extracted in the number plate extraction phase. From the entire input vehicle image, only the number plate area is extracted after detection and processed further in character segmentation. From the extracted number plate each character is isolated in character segmentation phase. After this the segmented characters are recognized in the character recognition phase. Extraction of number plate is a difficult task. Before the extraction of number plate area we have to detect that particular area from the whole vehicle body and when that area becomes visible we extract that portion. For a human being it is very easy to detect the number plate but for a machine it is really a difficult task and all further phases are also depending on the output of this phase. Therefore there should be accurate detection and extraction of number plate area from whole body of vehicle image. Essentially, the difficulty during the detection and extraction of number plate can be due to the following main reasons:-

1. Number plate is generally a small portion of the whole vehicle image so for a machine it is difficult to extract that accurate portion.

2. Number Plates exist on different location for different vehicles.

3. Weather conditions (fog, snow, rain) responsible for introducing image "noise".

4. Scene complexity (vehicle occlusion, background objects, focus, multiple objects etc.) introduce confusion.

5. Time of day (day and night) which affects lighting that result into contrast problems.

6. Wrong plate or camera position result into distortion.

7. Variation in styles, colors and formats of number plates. The difference in the size of number plates (aspect ratio), single versus multi-row, background graphics, dirty plates, light-on dark versus dark on light background, color of characters, font style, font size, font scripts, spacing between subsequent characters, number of lines in the plate, background color etc. increase difficulty during detection of number plate.
8. Mostly the detection is performed without prior knowledge of the number plate's location in the vehicle image.

9. In addition to unwanted characters sometimes a number plate may contain various frames and screws.

10. Uneven or low illumination, low resolution of the vehicle image, motion of vehicle, blurred image, distorted characters, reflection or shadows etc. effect the efficiency of detection and extraction of number plate.

This paper is organized in VI sections. Section II explains literature survey, Section III explain various phases of proposed number plate extraction method. Experimental result is shown in section IV. Conclusions and future work is given in Section V, References are given in section VI.

\section{LITERATURE SURVEY}

Ronak P Patel [1] proposed new algorithm for recognition number plate using Morphological operation and bounding box analysis for number plate extraction. Shan $\mathrm{Du}$ [2] presented a comprehensive survey on existing ANPR techniques by categorizing them according to the features used in each stage and compare them in terms accuracy, and processing speed. Najeem Owamoyo [3] proposed method for number plate extraction using Sobel filter and morphological operations. Divya Gilly [4] presented an efficient method for license plate detection by connected component analysis. Isack Bulugu [5] has proposed edge finding method to find the location of the plate. Rupali Kate [10] proposed algorithm based on a combination of morphological operation with area criteria tests for number plate localization. Hadi Sharifi [14] has study and evaluates some most important license plate detection algorithms and compared them in terms of accuracy, performance, complexity, and their usefulness in different environmental condition. This evaluation gives views to the developers or end-users to choose the most appropriate technique for their applications. The study and investigation show that the dynamic programming algorithm is the fastest and the Gabor transform is the most accuracy algorithm compared to other algorithms. Kumar Parasuraman and P.Vasantha Kumar [15] proposed algorithm for extracting the Plate region using edge detection algorithm and vertical projection method.

This paper presents a simple method for the extraction of number plate from the vehicle image based on morphological operations, thresholding and sobel edge detection and the connected component analysis.

\section{THE PROPOSED NUMBER PLATE EXTRACTION}

In this section the proposed number plate extraction method for Indian vehicles is represented. Input to the system is 
vehicle image that is acquired by digital camera and output is the actual number plate area. Flowchart of proposed algorithm is shown in figure 3 consists of following steps:

1) Input vehicle image

2) RGB to grayscale conversion

3) Contrast enhancement by using histogram equalization

4) Morphological opening and image subtraction operation

5) Image binarization using thresholding

6) Edge detection by sobel operator

7) Detection of candidate plate area by opening and closing operations

8) Extraction of actual number plate area

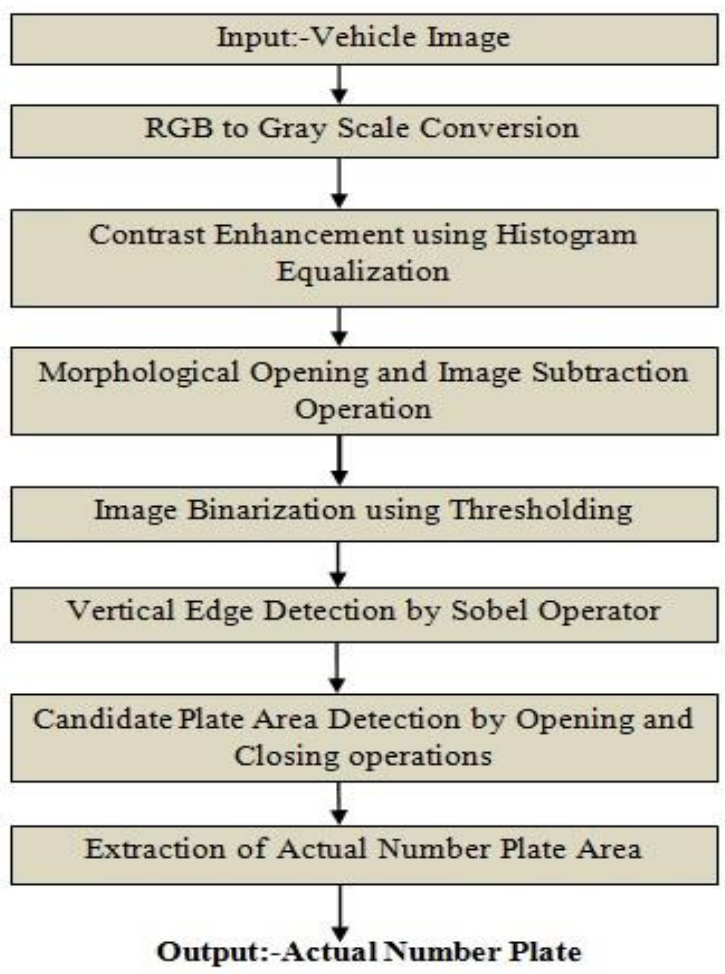

Fig 3: Flowchart of Proposed Method

\subsection{Image Acquisition}

The first step is to acquire the image of vehicle. Image is acquired by a high resolution digital camera. Images are acquired in different illumination conditions and in different background. Figure 4 shows the input vehicle image.

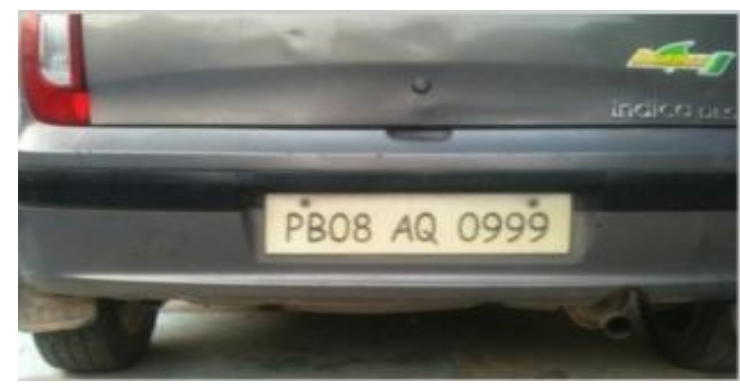

Fig 4: Input Image

\subsection{Pre-Processing}

Pre-processing is done on input vehicle image to enhance the visibility of image to improve the contrast of the image, enhance the processing speed and to reduce the noise in the image. In order to reduce the problem of low contrast and low quality in vehicle input images, images are enhanced by using histogram equalization on gray scale image. In Pre-Processing firstly RGB image is converted into gray scale image and then contrast enhancement is done with histogram equalization. Figure 5 shows the gray scale image, figure 6 show image histogram before contrast enhancement, and figure 7 shows image histogram after contrast enhancement using histogram equalization and figure 8 shows gray scale image after contrast enhancement.

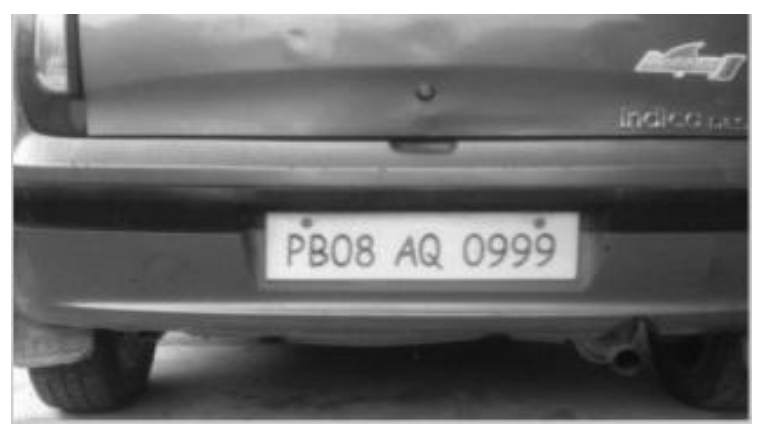

Fig 5: Gray Scale Image

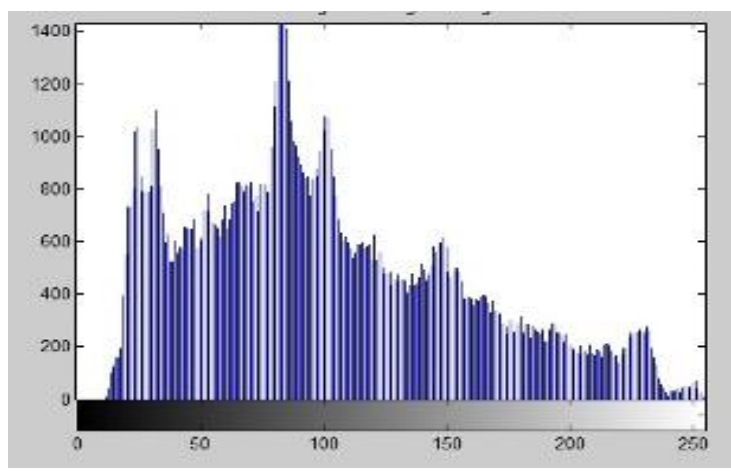

Fig 6: Histogram of gray scale image before Contrast Enhancement

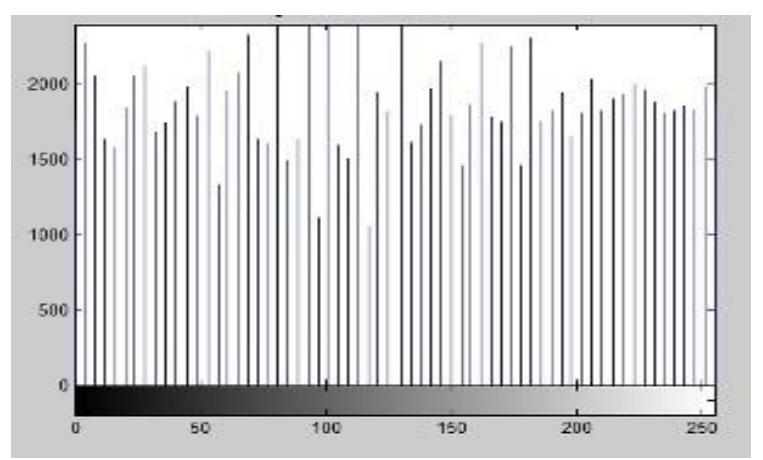

Fig 7: Histogram of gray scale image after Contrast Enhancement using Histogram Equalization 


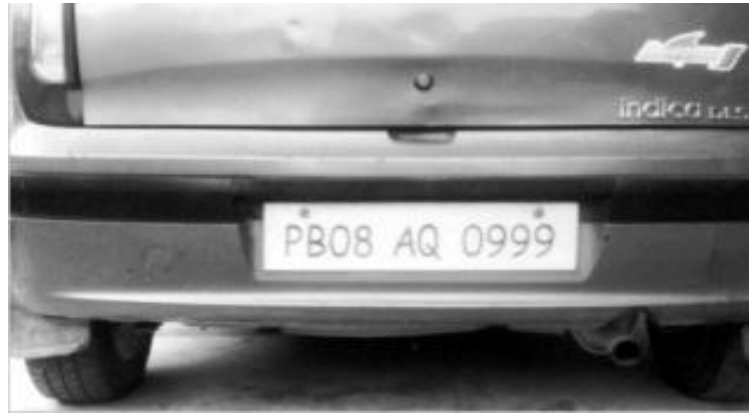

Fig 8: Gray scale image after Contrast Enhancement

\subsection{Morphological Opening and Image Subtraction}

Morphological opening operation is performed on the enhanced gray scale image by using structuring element and then subtracted from enhanced gray scale image. Figure 9 shows the result of opening operation on enhanced gray scale image using disk and figure 10 shows the result of subtraction between these two images.

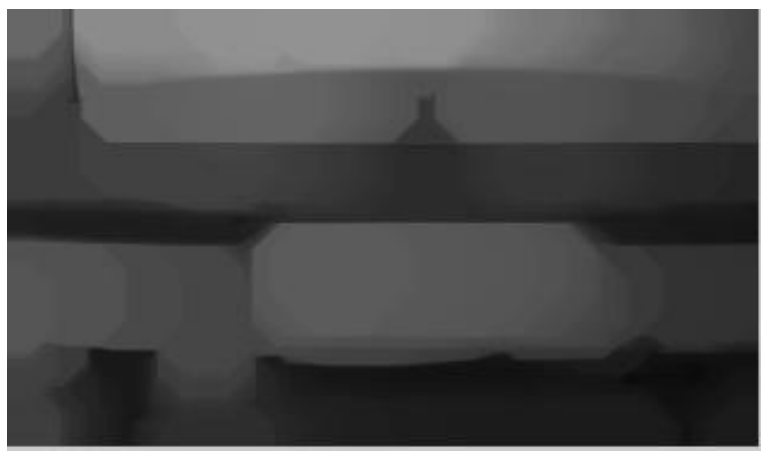

Fig 9: Opening effect using disk

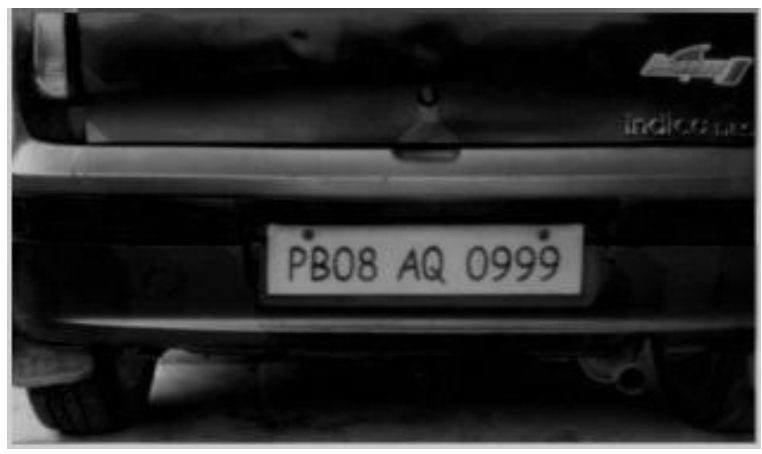

Fig 10: Image Subtraction

\subsection{Image Binarization}

In this operation gray scale image is converted into black and white image by calculating threshold level using Otsu's method. Firstly level of threshold is computed using graythesh function and then according to the calculated threshold level gray scale image is converted into binary image by using im 2 bw. Figure 11 shows binarized image.

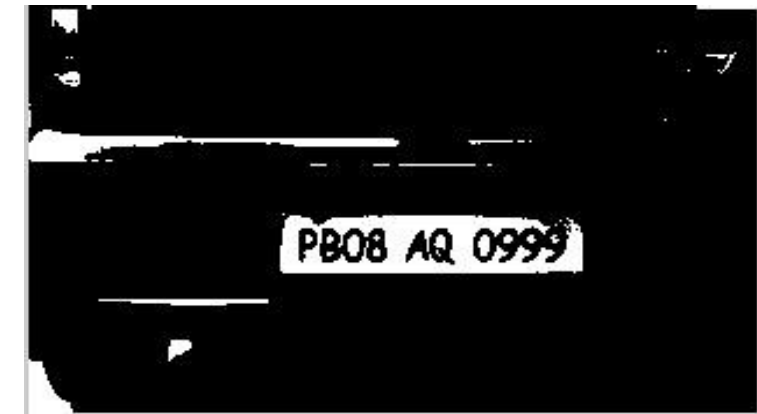

Fig 11: Binarized Image

\subsection{Vertical Edge Detection by Sobel Operator}

Vertical edge detection is done by sobel operator and result of applying sobel operator is shown in figure 12 as follow:-

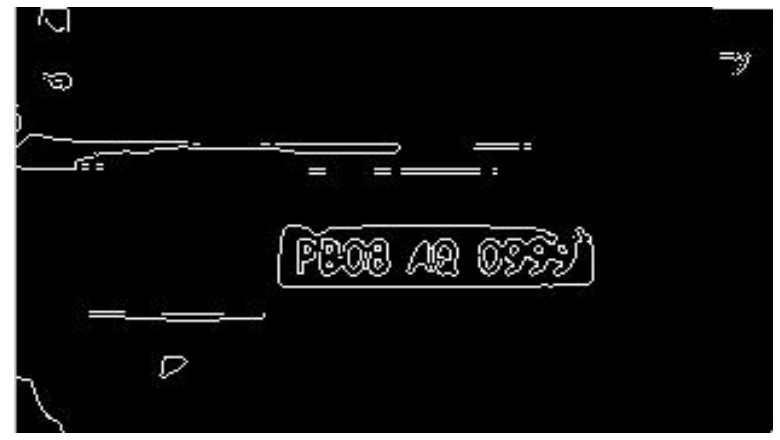

Fig 12: Vertical Edge Detection by Sobel operator

\subsection{Detection of Candidate Plate Area by Morphological Opening and Closing \\ Operations}

Morphological operations are mainly used to remove unwanted objects in the image. In this step firstly dilation operation is used and after this holes are filled by using MATLAB toolbox imfill function. The result of dilation operation and after filling holes function is shown in figure 13 and 14 respectively. After this morphological opening and erode operations are used for the detection of candidate plate area and its result in shown in figure 15.

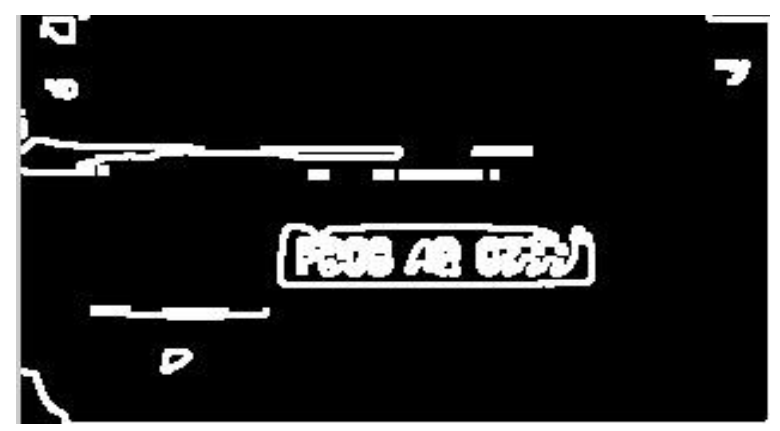

Fig 13: Result of Morphological Dilation Operation 


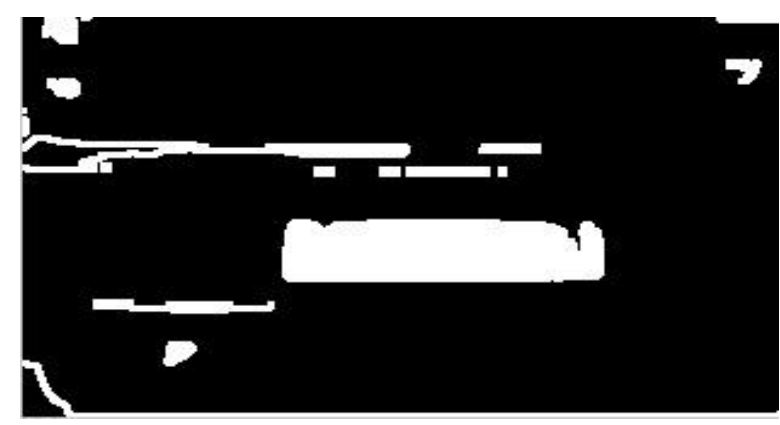

Fig 14: Image after filling holes

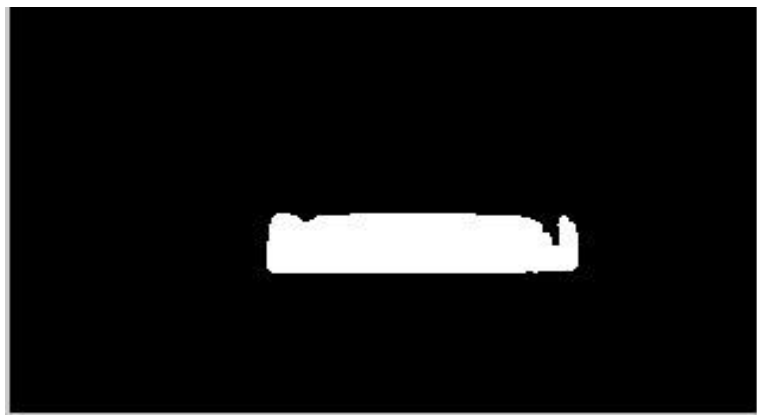

Fig 15: Number Plate Area Detection

\subsection{Extraction of True Number Plate Area}

After the detection of number plate area, the actual number plate area is extracted after finding the row and column indices of plate area by connected component analysis. Figure 16 shows the actual extracted number plate.$$
\text { PBO8 AQ } 0999
$$

Fig 16: Extracted True Number Plate

\section{EXPERIMENT RESULTS}

This proposed number plate extraction method work well for all types of vehicle images (png, jpeg, jpg, tif, tiff, bmp etc). Total 40 vehicle's images are used for testing. Images are taken in different illumination as well as background conditions. They are of different colors and different sizes. The images are taken from different distances relative to camera. The result of proposed method for number plate extraction is shown in table 1.This method is implemented in MATLAB 7.8.0.

Table 1: Result of Number Plate Extraction

\begin{tabular}{|l|l|l|}
\hline $\begin{array}{l}\text { Total } \\
\text { Vehicles } \\
\text { Images }\end{array}$ & $\begin{array}{l}\text { Successfully } \\
\text { Extracted Number } \\
\text { Plates }\end{array}$ & $\begin{array}{l}\text { Success } \\
\text { Rate (\%) }\end{array}$ \\
\hline 40 & 35 & $36 / 40=90 \%$ \\
\hline
\end{tabular}

Reflection, deep shadows and dirty plates have an impact on number plate recognition work and true number plates could not extract correctly.

\section{CONCLUSION AND FUTURE WORKS}

A simple and efficient number plate extraction method is presented in this paper. The proposed method is mainly designed for real-time Indian vehicles number plate but it also works well for foreign number plates. By using method we have extracted number plate area from image of vehicle. This extraction process is tested over more than one number plates of vehicles under different illumination and various weather conditions i.e. daytime, night time, rainy days, cloudy, sunny etc and success rate achieved by using this method is good. In future, the extraction of number plate is done in video-based ANPR.

\section{REFERENCES}

[1] Ronak P Patel, Narendra M Patel and Keyur Brahmbhatt, "Automatic Licenses Plate Recognition", International Journal of Computer Science and Mobile Computing, April 2013.

[2] Shan Du, Mahmoud Ibrahim, Mohamed Shehata and Wael Badawy, "Automatic License Plate Recognition (ALPR): A State-of-the-Art Review", IEEE Transaction on Circuits and Systems for Video Technology, February 2013.

[3] Najeem Owamoyo, A.Alaba Fadele and Abimbola Abudu, "Number Plate Recognition for Nigerian Vehicles", Academic Research International, 2013.

[4] Divya Gilly and Dr. Kumudha Raimond, "License Plate Recognition- a Template Matching Method", International Journal of Engineering Research and Applications (IJERA), 2013.

[5] Isack Bulugu, "Algorithm for License Plate Localization and Recognition for Tanzania Car Plate Numbers", International Journal of Science and Research (IJSR), 2013.

[6] Chen Chunyu, Wang Fucheng and Zhang Chen, "Application of image processing to the vehicle license plate recognition", Proceedings of the 2nd International Conference on Computer Science and Electronics Engineering (ICCSEE), 2013.

[7] Suhan Lee, Jeonghwan Gwak and Moongu Jeon, "Vehicle Model Recognition in Video", International Journal of Signal Processing, Image Processing and Pattern Recognition, 2013.

[8] Lukadev Meher, "Automatic License Plate Recognition in Real Time Videos using Visual Surveillance Techniques", ITSI Transactions on Electrical and Electronics Engineering (ITSI-TEEE), 2013.

[9] Aditya Acharya, and Sukadev Meher, Region Adaptive Unsharp Masking based DCT Interpolation for Efficient Video Intra Frame Up-Sampling”, IJCA Special Issue on International Conference (ICEDSP) February 2013.

[10] Rupali Kate, "Number Plate Recognition Using Segmentation", International Journal of Engineering Research and Technology (IJERT), November 2012.

[11] Lekhana G.C, R.Srikantaswamy, "Real Time License Plate Recognition System", International Journal of Advanced Technology \& Engineering Research (IJATER), July 2012.

[12] P.Mohan Kumar, P.Kumaresan and Dr.S.A.K.Jilani, "The Real Time Vehicle License Plate Identification System", International Journal of Engineering Research and Development, July 2012.

[13] Khalid W. Maglad, "A Vehicle License Plate Detection and Recognition System", Journal of Computer Science, 2012. 
[14] Hadi Sharifi Kolour, "An Evaluation of License Plate Recognition Algorithms", International Journal of Digital Information and Wireless Communications (IJDIWC), 2011.

[15] Kumar Parasuraman and P.Vasantha Kumar, "An Efficient Method for Indian Vehicle License Plate Extraction and Character Segmentation", IEEE International Conference on Computational Intelligence and Computing Research, 2010.
[16] S.Hamidreza Kasaei, S. Mohammadreza Kasaei and S. Alireza Kasaei, "New Morphology-Based Method for Robust Iranian Car Plate Detection and Recognition", International Journal of Computer Theory and Engineering, 2010.

[17] Manuel Vargas, "An Enhanced Background Estimation Algorithm for Vehicle Detection in Urban Traffic Scenes", IEEE Transactions on Vehicular Technology, 2010 . 